been shown not to be peritoneal mesotheliomata, since the clinical course and macroscopic appearances of these neoplasms can be very similar (Hourihane, 1964).

While necropsy and radiological surveys of other populations would be of great interest, and while the hazards of asbestos exposure are in need of more detailed analyses, the preventive and legal aspects of industrial, domestic, and environmental exposure require urgent consideration. Once pleural plaques have developed the dangers of supervening malignancy are great and the possibility of successful treatment is remote.

\section{Summary}

A close association between exposure to asbestos and the development of pleural plaques has been shown. A cause-andeffect relationship is probable. The most extensive lesions are radiologically detectable, and the disease asbestosis is then also present, despite the absence of any other radiological abnormality.

The sources of asbestos are considered and evidence is presented to suggest that there is a widespread non-industrial community exposure. Mesotheliomata are common in patients with pleural plaques, and it is suggested that there is a doseresponse relationship between the asbestos and the development of these neoplasms.

The question of a relationship between lung cancer and asbestos is left open, but the value of radiological surveys for pleural plaques is emphasized in any study of this problem.
Asbestos is not the sole cause of pleural plaques, but it is certainly the most common.

We should like to thank the physicians and surgeons of the London Hospital who allowed us to refer to cases under their care, and the registrars of the $x$-ray department who helped us to collect the radiological series. We are grateful to Professor I. Doniach, Dr. N. Lloyd-Rusby; and Dr. L. J. Rae for encouragement and assistance. We are indebted to Mr. Moss and the film store for their help in tracing a large number of films, to Miss R. L. Cresswell for secretarial assistance, and to the photographic department for the illustrations.

\section{REFERENCES}

Doll, R. (1955). Brit. 7. industr. Med., 12, 81.

Elmes, P. C., McCaughey, W. T. E., and Wade, O. L. (1965). Brit. med. 7., 1,350 .

Gloyne, S. R. (1933). Tubercle (Lond.), 14, 445, 493, 550

(1938). In Silicosis and Asbestosis, edited by A. J. Lanza. Oxford Univ. Press, London.

Heard, B. E., and Williams, R. (1961). Thorax, 16, 264.

Hourihane, D. O'B. (1964). Ibid., 19, 268.

- (1965a). M.D. Thesis, National University of Ireland. (1965b). Ann. N.Y. Acad. Sci., 132, 647.

Hurwitz, M. (1961). Amer. 尹. Roentgenol., 85, 256.

Kiviluoto, R. (1950). Acta radiol. (Stockh.), Suppl. No. 194.

Lawson, J. P. (1963). Clin. Radiol., 14, 414.

Selikoff, I. J., Churg, J., and Hammond, E. C. (1964). F. Amer. med. Ass., 188, 22.

Ass., 188, 22. (1952). Amer. F. Roentgenol., 67, 375.

Thomson, J. G. (1966). Ann. N.Y. Acad. Sci. In press.

Kaschula, R. O., and MacDonald, R. R. (1963). S. Afr. med. F., 37, 77.

Wagner, J. C., Sleggs, C. A., and Marchand, P. (1960). Brit. F. industr. Med., 17, 260.

\title{
Effect of Aggregating Agents on the Electrophoretic Mobility of Human Platelets
}

\author{
J. R. HAMPTON,* M.A., B.M., M.R.C.P.; J. R. A. MITCHELL, † M.D., D.PHIL., M.R.C.P.
}

Brit. med. F., 1966, 1, 1074-1077

In the presence of very low concentrations of adenosine diphosphate (A.D.P.), adenosine triphosphate (A.T.P.), 5-hydroxytryptamine (5-HT), and the adrenalines (Gaarder et al., 1961 ; Mitchell and Sharp, 1964) platelets in a citrated plasma system adhere together to form aggregates, but the mechanism of this type of aggregation is unknown. Platelets carry a negative surface charge (Abramson, 1928), and they would normally be expected to repel each other; during aggregation the electrostatic forces must be overcome. The surface charge on cells can be measured by determining their electrophoretic mobility, and we considered that a study of the effect of the various aggregating agents on platelet charge might yield information on the nature of the clumping process. This paper reports the results of these studies.

\section{Methods}

Blood samples were obtained from healthy volunteers and from medical and surgical patients who did not have acute inflammatory conditions. Platelets from subjects with a variety of inflammatory states (pneumonia, phlebitis, pericarditis, and temporal arteritis) and with recent myocardial infarctions or pulmonary emboli behave abnormally and will be described separately (Hampton and Mitchell, 1966b).

Platelet-rich and platelet-poor citrated plasma were prepared, and the platelets were brought into contact with glass, as described previously (Hampton and Mitchell, 1966a). Platelet-rich citrated plasma was diluted 1 in 10 with plateletpoor citrated plasma or with the other media specified below, and the electrophoretic mobility of platelets was measured in the horizontal capillary apparatus developed by Bangham et al. (1958). All measurements were made at $25^{\circ} \mathrm{C}$. with a potential gradient of $2.66 \mathrm{~V} / \mathrm{cm}$. The mobilities are expressed as $\mu / \mathrm{sec} . / \mathrm{V} / \mathrm{cm}$.

\section{Effect of A.D.P. and Noradrenaline on Mobility}

The post-contact mobility of platelets in a platelet-rich! platelet-poor citrated plasma mixture was measured. A.D.P. or noradrenaline (both from Sigma) was dissolved in $0.85 \%$

\footnotetext{
* Junior Lecturer, Department of the Regius Professor of Medicine, the Radcliffe Infirmary, Oxford.

+ First Assistant, Department of the Regius Professor of Medicine, the Radcliffe Infirmary, Oxford.
} 
sodium chloride solution, and added to the plasma system. The mixture was incubated at $25^{\circ} \mathrm{C}$. for 10 minutes and the platelet mobility was again measured. A range of concentrations of the two agents was tested, and a biphasic change in mobility was found. At low concentrations both agents produced a rise in platelet mobility ; in the group of patients used for this study the maximum increase in mobility was observed with $0.05 \mu \mathrm{g}$. of A.D.P. and noradrenaline per $\mathrm{ml}$. When the concentration of the agents was increased 10 -fold to 0.5 $\mu \mathrm{g} . / \mathrm{ml}$. they were found markedly to reduce platelet mobility (Fig. 1).

The time course of this change in mobility was determined by serial measurements at 10-minute intervals. Fig. 2 shows the results obtained with A.D.P. and noradrenaline, 0.05 $\mu \mathrm{g} . / \mathrm{ml}$. The maximum increase in mobility occurred 10 minutes after the agents had been added to the plasma mixture, and thereafter mobility gradually decreased. In all cases it had returned to its initial level at 60 minutes, and further additions of A.D.P. and noradrenaline at this time produced the same sequence of changes. The decrease in mobility produced by higher concentrations of the agents showed a similar timecourse, the maximum reduction in mobility with $0.5 \mu \mathrm{g}$. of A.D.P. and noradrenaline per $\mathrm{ml}$. occurring at 10 minutes.

Fig. 2 also shows that the manipulations involved in these measurements do not contribute to the observed changes, for when saline was substituted for the active agents there was no significant difference between the starting value and any of the subsequent values, whereas with $0.05 \mu \mathrm{g}$. of A.D.P. and noradrenaline per ml. the difference between the initial mobility
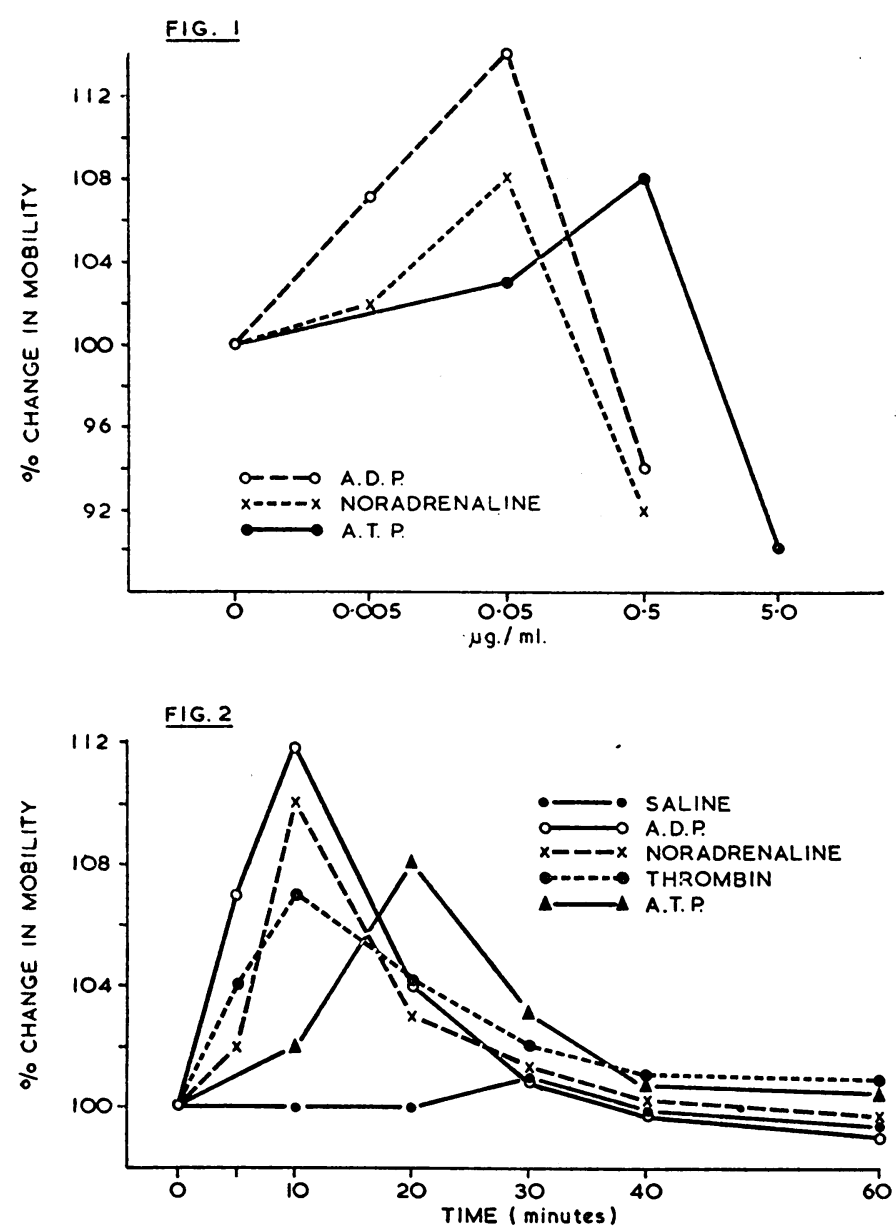

Fig. 1.- Mean percentage change in platelet electrophoretic mobility 10 minutes after the addition of A.D.P., A.T.P., and noradrenaline to platelet-rich/platelet-poor citrated plasma mixture ( $n=6$ for each agent) FIG. 2.-Mean time-course of percentage change in platelet electrophoretic Fig. 2.- Mean time-course of percentage change in platelet electrophoretic
mobility induced by A.D.P. $0.05 \mu \mathrm{g} . / \mathrm{ml}$. noradrenaline $0.05 \mu \mathrm{g} . / \mathrm{ml}$.,
A.T.P. $0.5 \mu \mathrm{g} . / \mathrm{ml}$., and thrombin $0.01 \mathrm{unit} / \mathrm{ml}$. ( $\mathrm{n}=5$ for each agent). and the mobility after 10 minutes' incubation was highly significant $(\mathrm{P}<0.001)$.

\section{Effect of Adenosine Triphosphate on Mobility}

When increasing concentrations of A.T.P. (Sigma) were added to platelet-rich and platelet-poor citrated plasma mixtures a similar biphasic response with first an increased and then a decreased mobility was induced. The concentrations of A.T.P. required were, however, 10 times higher than those of A.D.P. or noradrenaline: the maximum increase in mobility was produced by $0.5 \mu \mathrm{g} . / \mathrm{ml}$. and a significant decrease in mobility was produced by $5 \mu \mathrm{g}$./ml. (Fig. 1). The differences between the starting mobility values and those observed after the addition of A.T.P. 0.5 and $5 \mu \mathrm{g} . / \mathrm{ml}$. were highly significant $(\mathrm{P}<0.001)$.

The time-course of the changes in mobility was slower than with A.D.P. and noradrenaline, the maximum increase or decrease in mobility occurring after 20 minutes' incubation (Fig. 2). However, if human white blood cells were added to the platelet-rich and platelet-poor citrated plasma mixture the maximum change occurred after 10 minutes' incubation with A.T.P. This observation is in accordance, "yith the evidence that A.T.P. is converted by white blood cells into A.D.P., which is the active aggregating agent (Harrison et al., 1966).

\section{Effect of Thrombin on Mobility}

Increasing concentrations of thrombin (Fibrindex, Ortho) produced the same biphasic response. The maximum increase in mobility occurred at a final concentration of $0.01 \mathrm{unit} / \mathrm{ml}$. and a significant increase was still detectable at $0.001 \mathrm{unit} / \mathrm{ml}$. The concentration of thrombin had to be increased to 1 unit $/ \mathrm{ml}$. before a significant decrease in mobility was observed. Thrombin therefore differs from A.D.P., A.T.P., and noradrenaline in that there is a 100-fold rather than a 10-fold difference between the concentration causing the maximum increase and that causing a significant decrease in mobility. Like A.D.P. and noradrenaline, but unlike A.T.P., the maximum change in mobility occurred after 10 minutes' incubation (Fig. 2).

\section{Effect of Collagen}

Fresh human Achilles tendon was obtained at necropsy, cut into small pieces, and ground up with a small quantity of saline. The resulting mixture was centrifuged to remove particulate matter, and the supernatant was stored at $-15^{\circ} \mathrm{C}$. until required. It was found that this extract changed the mobility of platelets in the same biphasic way as the other aggregating agents : the concentrated solution caused a decrease, and a 10 -fold dilution the maximum increase, in mobility. The maximum change in mobility occurred after 10 minutes' incubation with all concentrations of the extract, and, as with the other agents, the mobility returned to the initial value within one hour.

\section{Effect of 5-Hydroxytryptamine}

Saline solutions of 5-HT, giving final concentrations of from 0.03 to $1 \mu \mathrm{g} . / \mathrm{ml}$., were added to platelet-rich/platelet-poor citrated plasma mixtures. Measurements of platelet mobility were made as soon as possible (two to three minutes) after their addition and repeated at 10-minute intervals over an hour. No consistent change in mobility with any concentration could be detected. 


\section{Effect of Heparin}

Platelet-rich/platelet-poor plasma mixtures were prepared from six blood samples collected into heparin, the final concentration of heparin varying from 1 to 50 units $/ \mathrm{ml}$. The change in mobility observed with A.D.P. or noradrenaline $0.005,0.05$, and $0.5 \mu \mathrm{g} . / \mathrm{ml}$. did not differ significantly from that of our standard platelet-rich/platelet-poor citrated plasma mixtures prepared from the same blood samples.

\section{Substitutes for Platelet-poor Plasma}

Methods of investigating platelet aggregation such as the optical density technique (Born, 1962) require a concentration of platelets similar to that found in platelet-rich plasma. The problems of resuspending platelets in other media have made it difficult to establish which plasma constituents are necessary for the aggregation process. Since a 1-in-10 dilution of platelet-rich citrated plasma suffices for the measurement of surface charge, it is possible to vary the composition of the diluting medium. A variety of solutions were used in an attempt to establish what factors were required for the changes which A.D.P. a noradrenaline had been shown to induce in the surface charge of platelets suspended in their own plasma.

(a) Inorganic Salts and Albumin.-Platelet-rich citrated plasma was diluted 1 in 10 with $0.85 \%$ saline. No change in platelet mobility could be detected when A.D.P. and nor-
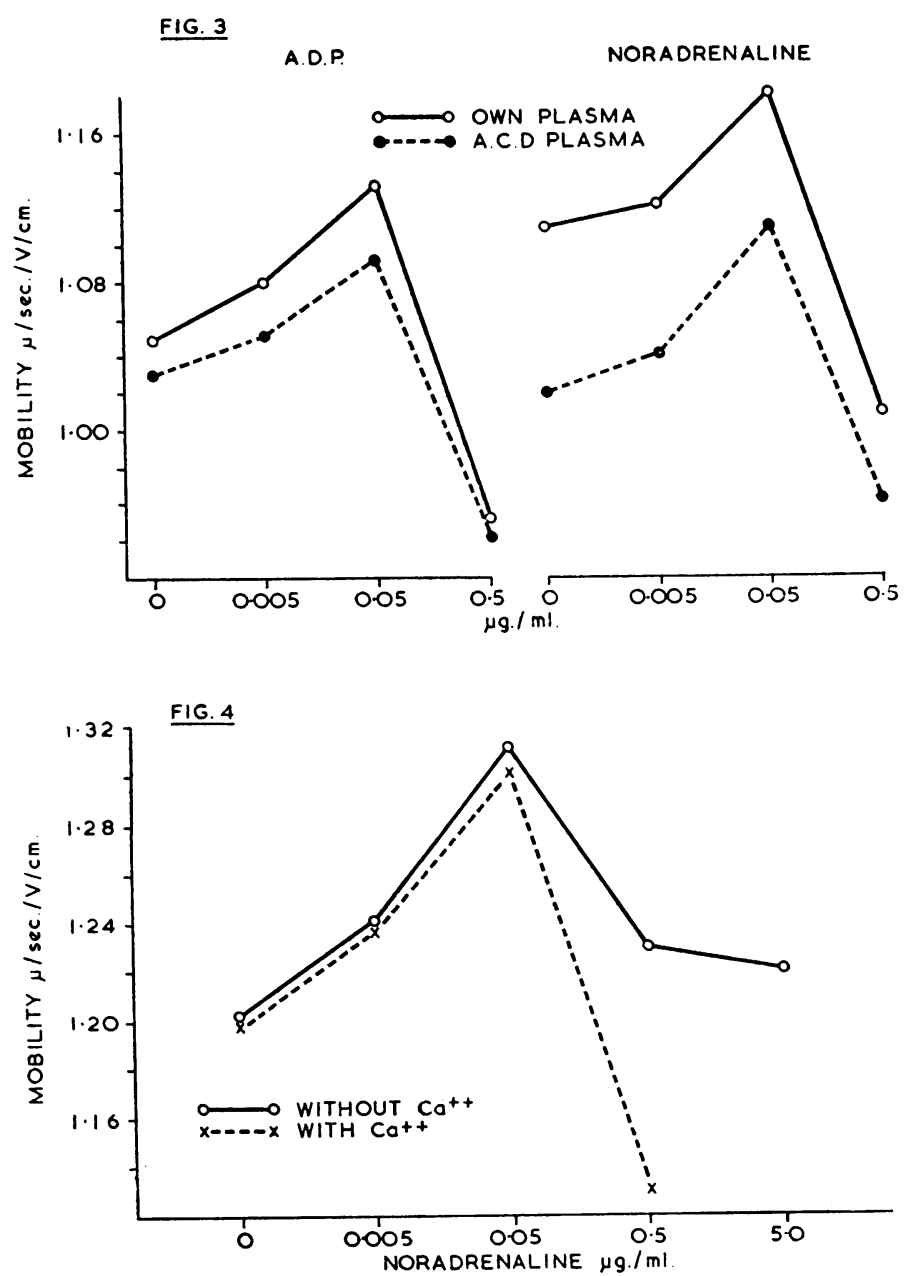

FIG. 3.-Mean change in platelet electrophoretic mobility induced by A.D.P. and noradrenaline with platelets suspended in their own plateletpoor plasma and in stored acid-citrate-dextrose plasma $(n=3)$. FIG. 4.Mean change in platelet electrophoretic mobility induced by noradrenaline with platelets suspended in reconstituted dried human plasma without added calcium and with added calcium, $0.025 \mathrm{M}$ in final mixture $(n=2)$. adrenaline, 0.5 and $0.05 \mu \mathrm{g} . / \mathrm{ml}$., were added to the mixture. Tris-buffered saline was likewise ineffective as a suspending medium. When $0.05 \mu \mathrm{g}$. of A.D.P. per ml. was added to a mixture containing platelet-rich citrated plasma in $75 \%$ platelet-poor citrated plasma and $25 \%$ saline a significant increase in mobility was produced, although it was smaller than that with the normal platelet-rich/platelet-poor citrated plasma mixture. If the platelet-rich citrated plasma was diluted with a mixture of equal volumes of platelet-poor citrated plasma and saline the addition of A.D.P. caused no change in mobility. A modified Krebs solution, buffered with bicarbonate and containing calcium, magnesium, potassium, phosphate, and glucose in addition to sodium chloride, was used instead of saline. A.D.P. caused a detectable increase in mobility when platelet-rich citrated plasma was diluted with a mixture of $25 \%$ platelet-poor citrated plasma and $75 \%$ of this solution; but when platelet-rich citrated plasma was diluted with the solution alone it had no effect, and A.D.P. also failed to cause any change in surface charge if $5 \mathrm{~g}$. per $100 \mathrm{ml}$. of bovine albumin was added to the modified Krebs solution.

(b) Old Human Plasma.-Acid-citrate-dextrose blood between two and three weeks old was obtained from the blood-transfusion service and plasma free from cellular constituents was obtained by centrifugation. One-in-ten dilutions of platelet-rich citrated plasma in this plasma were prepared. Though the mean post-contact mobility in this system was significantly lower than that of the same platelets suspended in their own fresh platelet-poor citrated plasma (seven experiments, mean difference $0.03 \mu / \mathrm{sec} . / \mathrm{V} / \mathrm{cm}$., $\mathrm{P}=0.05$ ), the biphasic pattern of the dose response with A.D.P. and noradrenaline was similar whether the diluent was fresh or stored plasma (Fig. 3).

(c) Dried Human Plasma.-Dried human plasma was dissolved in distilled water, and the solution was then filtered twice. One-in-ten dilutions of platelet-rich citrated plasma were made in this solution, and it was found that $7 \mathrm{~g}$. of dried plasma in $100 \mathrm{ml}$. of water gave post-contact mobilities comparable to those obtained when fresh platelet-poor citrated plasma was used as the suspending medium. Dose-response curves for A.D.P. and noradrenaline were determined with this mixture. An increase in mobility similar in magnitude to that observed in platelet-poor citrated plasma was produced by $0.05 \mu \mathrm{g}$. of the agents per ml., but increasing the concentration up to $50 \mu \mathrm{g} . / \mathrm{ml}$. no longer gave the expected reduction in mobility. The usual biphasic pattern seen in platelet-poor citrated plasma was restored by adding calcium chloride to give a final concentration of $0.025 \mathrm{M}$. As this concentration of calcium chloride was sufficient to cause the mixture to clot, and as heparin does not affect the change in mobility produced by the agents (see above), these studies were performed in the presence of heparin 1 unit/ml. The mean results of two experiments with noradrenaline are shown in Fig. 4.

(d) Human Fibrinogen.-Some constituent of dried plasma, absent from the albumin Krebs saline solution, appeared to be necessary for the changes in electrophoretic mobility which had been observed. Fibrinogen has been suggested as a co-factor in platelet aggregation (McLean et al., 1964), and we therefore tested a mixture of human fibrinogen (Kabi) and citrate-saline-calcium chloride, the system providing $250 \mathrm{mg}$./ $100 \mathrm{ml}$. of fibrinogen and $0.025 \mathrm{M}$ calcium chloride in the final mixture. When A.D.P. was added to platelet-rich citrated plasma diluted 1 in 10 with this system no change in mobility was found. However, when fibrinogen was added to the modified Krebs saline solution to give a final concentration of $250 \mathrm{mg} . / 100 \mathrm{ml}$. in the final platelet-rich citrated plasmaKrebs saline mixture, the normal biphasic change in mobility was produced by A.D.P. However, no change in mobility could be produced by the addition of noradrenaline or thrombin to the mixture. We must conclude that, while the simple inorganic constituents of the Krebs saline plus glucose and fibrinogen can replace plasma as a diluting medium when 
A.D.P. is used to alter mobility, other factors must be involved in the changes in mobility produced by noradrenaline and thrombin.

\section{Effect of Ethylenediamine Tetra-acetic Acid (E.D.T.A.)}

A platelet-rich/platelet-poor citrated plasma mixture was prepared, and varying concentrations of dipotassium E.D.T.A. were added. The effect of A.D.P. and noradrenaline on platelet mobility was then compared with their effect on a platelet-rich/platelet-poor citrated plasma mixture to which saline had been added instead of E.D.T.A. The increase in mobility usually produced by $0.05 \mu \mathrm{g} . / \mathrm{ml}$. of both agents was completely abolished by a final concentration of E.D.T.A. of $10^{-2} \mathrm{M} ; 10^{-3} \mathrm{M}$ allowed a small increase in mobility, while with $10^{-4} \mathrm{M}$ the increase in mobility was the same as in the parallel control series. The reduction in mobility usually produced by $0.5 \mu \mathrm{g}$. of A.D.P. and noradrenaline per ml. was, however, completely abolished by $10^{-3} \mathrm{M}$ E.D.T.A., substantially reduced by $10^{-4} \mathrm{M}$, and not until the E.D.T.A. concentration was reduced to $10^{-5} \mathrm{M}$ did the inhibitory effect disappear. It thus appears that the process which leads to a decrease in platelet mobility in the presence of A.D.P. or noradrenaline $0.5 \mu \mathrm{g} . / \mathrm{ml}$. is ten times more sensitive to E.D.T.A. than the increase in mobility produced by $0.05 \mu \mathrm{g}$. of the aggregating agents per $\mathrm{ml}$.

\section{Discussion}

We have shown that the agents which produce platelet aggregation also produce a biphasic change in platelet surface charge, low concentrations of the agents increasing platelet mobility and higher concentrations reducing mobility. Although we have not observed aggregates in the electrophoresis apparatus where the plasma column is stationary, the concentrations which produced a reduction in mobility are similar to those which have been shown (Gaarder et al., 1961 ; Mitchell and Sharp, 1964) to cause aggregation in a stirred system. Since the mutual repulsion between platelets must be overcome in the aggregation process, a reduction of surface charge by the clumping agents would provide a mechanism by which these electrostatic forces could be minimized. The increase in mobility produced by smaller concentrations of the agents is, however, less easy to understand. A.D.P. is negatively charged, and it is known that it can be taken up by platelets; it seems possible that it is adsorbed on to the platelet surface and causes an increase in the net negative charge. Noradrenaline is, however, positively charged, and could not produce an increase in mobility by simple adsorption. The way in which complex materials such as thrombin and collagen produce an increase in surface charge is also obscure.

Whatever the mode of origin of the change in mobility, the process must be an active and dynamic one, for both the increase and the decrease in mobility reach a maximum intensity at a time which is determined by the particular agent used. There is then a slow return to the initial mobility value, which is reached 60 minutes after adding the agents, and the sequence of changes can be repeated if fresh agent is added. The reversible nature of the change would accord well with the pattern of aggregation found with A.D.P., where disaggregation is always observed with low concentrations of the agent but not with the pattern found with A.T.P., noradrenaline, and collagen, where the aggregates, once formed, do not usually disperse. Thus, although there are many similarities between the changes in mobility and platelet aggregation, there are equally striking discrepancies.

A.D.P. can produce an increase in the surface charge of platelets in the presence of inorganic ions, glucose, and fibrinogen, whereas noradrenaline and thrombin require some other factor, which can be provided by adding dried plasma. Two different mechanisms may therefore be involved, and we shall present further evidence that this is so (Hampton and Mitchell, 1966c). Nevertheless, the final effect of the two types of agent is closely linked, and it is possible that noradrenaline, having been taken up by the platelet, releases A.D.P., which is retained on the platelet surface and affects charge (O'Brien, 1964).

The increase and decrease in mobility with aggregating agents are both calcium-dependent; the latter change appears to be more calcium-demanding, since it is abolished by one-tenth of the concentration of E.D.T.A. needed to abolish the former. It is possible, therefore, that the increase in surface charge represents a state in which the platelet is prepared for the adsorption of calcium ; at a critical concentration of the agents, calcium adsorption occurs and the surface charge is reduced.

As a hypothesis on which further work can be based, we suggest that when exogenous agents produce aggregation in vitro A.D.P. is either adsorbed on to the platelet surface from without or released on to the surface from within. As the adsorbed A.D.P. builds up, surface charge and hence electrophoretic mobility are increased. At a critical concentration of surface A.D.P. which provides optimal charge-density and spatial arrangement, calcium adsorption occurs. The ensuing reduction in surface charge diminishes the repulsive forces between platelets, and if the system is stirred so as to bring the platelets together aggregation will occur.

\section{Summary}

The surface charge of human blood platelets has been investigated by measurement of their electrophoretic mobility. Adenosine diphosphate, adenosine triphospate, noradrenaline, thrombin, and an extract of collagen all cause a biphasic change in mobility: in concentrations which would cause aggregation in a stirred system there is a decrease in mobility, but with lower concentrations there is an increase. With all concentrations of these agents the maximum change occurs after 10 to 20 minutes, and in all cases this change is spontaneously reversible.

It has been found that adenosine diphosphate will induce these changes in platelets suspended in a solution containing inorganic ions, glucose, and fibrinogen, but other factors are apparently necessary for the noradrenaline and thrombin induced effects.

The decrease in mobility with higher concentrations of these agents is more sensitive to ethylenediamine tetra-acetic acid than is the increase caused by lower concentrations.

We are grateful to Sir George Pickering for advice and encouragement ; to Dr. A. D. Bangham for helpful discussions of problems of cell electrophoresis ; and to Dr. J. Grant, of the Oxford Regional Blood Transfusion Service, for supplies of human blood products.

\section{REFERENCES}

Abramson, H. A. (1928). 7. exp. Med., 47, 677.

Bangham, A. D., Flemans, R., Heard, D. H., and Seaman, G. V. F. (1958). Nature (Lond.), 182, 642.

Born, G. V. R. (1962). F. Physiol. (Lond.), 162, 67P.

Gaarder, A., Jonsen, J., Laland, S., Hellem, A., and Owren, P. A. (1961). Nature (Lond.), 192, 531.

Hampton, J. R., and Mitchell, J. R. A. (1966a). Ibid., 209, 470.

- (1966b). Brit. med. f., 1, 1078.

- (1966c). Nature (Lond.). In press.

Harrison, M. J. G., Emmons, P. R., and Mitchell, J. R. A. (1966). Thromb. Diathes. haemorrh. (Stuttg.). In press.

McLean,' J. R., Maxwell, R. E., and Hertler, D. (1964). Nature (Lond.), 202, 605 .

Mitchell, J. R. A., and Sharp, A. A. (1964). Brit. f. Haemat., 10, 78.

O'Brien, J. R. (1964). f. clin. Path., 17, 275. 